\title{
Le labyrinthe médiatique du conteur d'aujourd'hui
}

\section{Bruno de la Salle}

\section{(2)enEdition}

\section{Journals}

Édition électronique

URL : http://journals.openedition.org/pa/181

DOI : $10.4000 /$ pa. 181

ISSN : 2273-0362

\section{Éditeur}

Université Lumière Lyon 2

\section{Édition imprimée}

Date de publication : 15 janvier 2009

Pagination : 10-20

ISBN : 978-2-912868-62-6

ISSN : 1634-7706

Référence électronique

Bruno de la Salle, «Le labyrinthe médiatique du conteur d'aujourd'hui », Parcours anthropologiques [En ligne], 7 | 2009, mis en ligne le 02 juillet 2013, consulté le 04 mai 2019. URL : http:// journals.openedition.org/pa/181; DOI : 10.4000/pa.181 


\title{
Le labyrinthe médiatique du conteur d'aujourd'hui
}

\author{
Bruno de la Salle
}

On sait bien que raconter une histoire est très simple, il suffit de pouvoir parler à quelqu'un d'autre, c'est pourquoi la narration orale est un art très ancien. Cette antériorité peut la faire considérer comme primitive et pourtant, bien que concurrencée par l'usage du livre ou d'objets numériques, elle est encore pratiquée aujourd'hui. Elle répond à des besoins profonds et récurrents, et nécessite des conditions spécifiques - tout particulièrement une maîtrise du maniement de la parole.

La question de la conservation, et donc de la mémorisation, est demeurée et demeure primordiale. Il est apparu à tous qu'il fallait se souvenir de l'histoire, des événements, des inventions, des connaissances, et surtout des valeurs, pour les retenir et les transmettre. Il fallait aussi se souvenir des conditions dans lesquelles ces paroles avaient été formulées, puisqu'on le sait, elles avaient été largement déterminées par le contexte dans lequel elles avaient été prononcées. Enfin, le but ultime de ces tentatives de conservation a été de transmettre le vivant et, à défaut d'y parvenir, d'au moins laisser des traces de paroles qui y reconduisent. C'était un moyen de transmission simple, direct, accessible à tous et qui ne nécessitait que les virtualités d'un corps et d'un esprit humains. Elle s'exerçait le plus souvent au cours d'un rassemblement qui s'inscrivait dans un temps et un espace géographique communs. Elle était polysémique, dans le sens où elle offrait un même récit à tous les membres d'une communauté, tout en permettant à chacun d'y trouver une réponse à son besoin propre. Elle était conçue pour être répétée et transmise tout au cours de la vie et pendant des générations. Elle nécessitait un apprentissage évident, puisque l'une de ses principales fonctions était la transmission de la pensée et du langage. Cet apprentissage prenait évidemment en compte, et en tout premier lieu, le sens du message à transmettre, mais aussi la nature de ses destinataires et les circonstances dans lequel il était délivré. Au sein de cette oralité étaient ainsi produites des œuvres humaines élaborées, mais adaptables aux instants et aux lieux où elles étaient échangées.

Il y a eu, plus tard, d'autres moyens de raconter - et d'abord l'écriture qui a engendré la littérature. Ce nouveau moyen de communication 
apportait à ses utilisateurs la possibilité d'échapper au temps - ou du moins de le suspendre, d'isoler le concepteur de la pression de la performance orale et de son public et, par là, lui donnait l'avantage de pouvoir planifier son message.

L'écriture a d'abord été produite, conservée et diffusée sur des matériaux d'origine végétale, minérale ou animale et depuis plusieurs siècles aussi, sur du papier et par le moyen de l'imprimerie. Elle nécessitait l'accès à des outils spécifiques de l'écriture et de l'imprimerie et la connaissance approfondie du maniement de ces outils.

La relation établie par l'imprimerie ne fut limitée ni par le nombre, ni par l'espace ou le temps. Elle permit ainsi de conserver, de mémoriser, de diffuser les productions mentales humaines sans autre limitation que celles imposées par la fabrication, le transport et la commercialisation de ce que l'on appelle le livre. Ce moyen établit une relation directe entre l'écrivain et son lecteur, sans pour autant leur permettre de se rencontrer dans la réalité de l'instant et celle de l'espace. On pourrait dire que le livre devint une pensée " hors sol », hors de soi. C'est là que se situa la limite de ses performances.

Au siècle dernier, apparut le cinéma qui prit le relais du conte et du théâtre... et du livre. Lui aussi bouleversa notre façon de raconter. L'histoire était parlante, visible et bougeante. Elle bougeait comme bougeaient les marionnettes, les ombres, les acteurs ou les danseurs dans les traditions anciennes en accompagnement de la narration parlée ou chantée. Mais en plus de ce mouvement, de ces couleurs, de ce son, de ce réalisme que l'image apportait, le film, comme le livre, se conservait et pouvait se multiplier. La puissance de son emprise fut et demeure telle que l'on peut dire que le film est devenu, plus encore que le livre, l'un des constituants principaux de notre imaginaire, et par conséquent de notre mémoire. Grâce à lui furent conservés des témoignages irremplaçables sur le passé de nos arts oraux anciens ainsi que les premiers essais de cet art naissant.

Il demeure, là aussi, que malgré sa puissance, le film laisse les deux interlocuteurs que sont celui qui s'exprime et celui qui perçoit, séparés presque inexorablement l'un de l'autre dans le temps et dans l'espace. C'est là aussi que se situe la limite de ses performances pourtant précieuses, nombreuses et fascinantes.

Pour raconter des histoires, il y a eu, enfin et très récemment, l'informatique. C'est une technique d'écriture, de formulation, de conservation, de reproduction, de communication d'informations qui ne peut plus déjà être considérée comme nouvelle tant son développement est exponentiel. Il est cependant certain que cette façon de communiquer renouvelle complètement les moyens d'expression et le transport de la pensée, du texte et de la parole.

Elle est incorporelle, le plus souvent intemporelle, sans lieu réel autre que la machine avec laquelle elle est produite, utopique aurait-on dit autrefois. Elle ouvre des champs de contacts presque inaccessibles sans elle et donne lieu à des possibilités de rencontre et de reproduction innombrables. Son usage n'est limité que par l'accès à l'électricité, aux réseaux de distribution par câbles ou satellites, et enfin et surtout à l'appareil informatique qui lui-même devient accessible à presque tous. 
Comme le livre, elle peut s'accompagner de sons et d'images fixes ou en mouvement. Elle est enfin, instantanée.

Il demeure encore que malgré les performances révolutionnaires et polymorphismes de l'informatique et de l'Internet de transport, de simultanéité entre l'expression et la perception du message, l'informatique ne peut prétendre à l'extrême qualité de la relation vivante, du corps à corps, de l'homme à homme vertical que permet le contact oral direct.

La relation vivante, en effet, ne nécessite aucun autre moyen que celui dont dispose tout être humain : une bouche, des oreilles, une mémoire, une intelligence, une sensibilité, une présence. Elle garantit une qualité de transmission inégalable. Elle demeure le moyen le plus performant dans la rencontre sensible entre plusieurs êtres. Elle pourrait redevenir, par sa rareté engendrée justement par la concurrence du livre, du cinéma et de l'informatique, l'événement exceptionnel et providentiel qu'elle a, en fait, toujours été. C'est pourquoi ce labyrinthe médiatique et quotidien que constituent ces outils, ces livres, ces films, ces messages numériques, ne doit être envisagé et emprunté aujourd'hui que comme une aide possible pour accéder à notre but de conteur qui est de raconter le mieux et le plus simplement possible.

\section{Un NOUVEAU CONTEUR}

Je suis ce que l'on appelle aujourd'hui un nouveau conteur, pas un conteur traditionnel au sens ethnologique du terme. Comme dit Homère, "Je n'ai pas eu de maître... ». Je ne suis pas né dans une communauté traditionnelle et orale, et je n'ai donc pas joui de son enseignement. J'ai lu et peut-être, dans mon enfance, entendu d'autres personnes qui, elles-mêmes avaient lu. Lorsque j'ai commencé à écrire puis à raconter, je ne prétendais qu'à poursuivre l'aventure des écrivains et poètes qui m'avaient précédé. En m'alignant sur ce modèle d'action du « toujours nouveau " qui caractérise la société occidentale, et donc sa littérature, je cherchais une nouvelle façon d'écrire, de m'exprimer d'une manière innovante pour répondre à des besoins du monde dans lequel je vivais et qui n'existaient plus, ou pas encore.

J'avais eu la chance, au cours de mes lectures d'enfance, d'être particulièrement touché par la beauté des textes narratifs traditionnels, et en premier lieu des contes, des légendes et des épopées. Mes premiers essais d'écriture, puis d'oralité, fortement influencés par le surréalisme, m'avaient mené vers le rêve. Et ce fut tout naturellement que je passai du rêve raconté oralement aux contes qui, eux aussi, étaient proches du rêve et s'exprimaient en paroles. Je comprenais qu'il y avait beaucoup à apprendre de cette tradition si longtemps méprisée. Je ne soupçonnais pas alors que, de surcroît, en me mettant à l'école de ces maîtres anonymes et discrets que furent les conteurs traditionnels oraux, j'entrais dans un monde, qui bien plus que de transmettre des contenus désormais écrits, nous invitaient surtout à redécouvrir, puis à acquérir, une véritable science de la parole, que l'on nommerait aujourd'hui Oralité. 
C'est par leur fréquentation que je découvris peu à peu que la parole était un moyen de représentation de l'invisible. Qu'elle montrait de l'invisible, étant elle-même invisible. Qu'elle permettait de représenter avec des signes sonores l'invisible de l'absence ou du disparu pour lutter contre l'oubli. Que cet invisible contenu dans les paroles était aussi invisible et aussi vital que l'oxygène, et qu'il participait peut-être plus encore que celui-ci à l'atmosphère nécessaire à l'humanité, et que sa raréfaction ou sa dégradation étaient un danger aussi grave que la plus grave des pollutions.

\section{LECTEUR-ÉCRIVAIN-WEBMASTER-}

\section{SCULPTEUR-AVIATEUR-CONTEUR}

Je suis conteur, mais je n'ai jamais quitté l'écriture et la lecture. Comment aurais-je fait? Nous sommes encore dans la lettre. C'est elle qui m'a conduit à l'oralité. Conteur, je suis un enfant de l'écriture et je ne pourrais pas prétendre à mon appartenance à l'humanité si je n'avais pas été élevé dans le livre.

À la suite de cet apprentissage, je me suis mis, et cela tout naturellement, à l'école de l'ordinateur, pour mieux écrire. Mieux écrire, cela voulait dire pour moi, ne pas faire de fautes d'orthographe, offrir à mes interlocuteurs une belle page propre, façonnée à ma discrétion; faire un document, peut-être même un livre, par moi-même. Je comptais, en m'exonérant des circuits de l'édition, découvrir ce que voulait dire s'exprimer par l'écriture imprimée directement et sans intermédiaire.

Il y avait d'autres avantages que le «mieux écrire ». Je les découvris peu à peu : le " copié-collé ", les mémorisations des "versions " si nombreuses que ma propre mémoire pouvait s'y perdre. Il y avait aussi cette providence d'informations directement accessibles, de corrections, d'ajouts... Il y avait également le moyen de compter, de composer mes mots et mes phrases, de les organiser visuellement pour mieux comprendre le mouvement de la parole et de la pensée et d'en rendre compte, de mettre l'écriture en mouvement. Et, à travers tous ces malaxages de textes, de dessiner des partitions de paroles qui, comme à l'origine de l'écriture, ne faisaient qu'aider à la conception, à l'enregistrement et la restitution de ma pensée.

Il y avait enfin tout ce que les progrès certains de ces machines me laissaient imaginer et en particulier ceux qui étaient entrepris sur la reconnaissance vocale qui, pour quelqu'un dont le but ultime était de parler, d'oraliser, laissait entrevoir quelques perspectives excitantes.

Mais, en revenant sur les souvenirs que m'ont laissé ces premières approches d'écriture numérique, il y avait ce pouvoir simple et primordial que nous offre l'ordinateur, qui est celui de voir les paroles. De les voir comme aucun autre outil ne l'avait aussi bien permis avant lui.

Lorsque j'ai commencé à écrire et, avant de passer à des œuvres orales, je me suis intéressé à la calligraphie ou plutôt à des calligrammes 
comparables à ceux qu'Apollinaire avait expérimentés. Je cherchais à donner à l'écrit une forme correspondant aux images que je voyais mentalement et dont pouvaient témoigner les signes dessinés que j'associais à mes mots. J'ai retrouvé cette approche plastique dans ce que permet l'ordinateur, mais différemment.

Ce n'était plus tant le dessin de l'image qui me paraissait important (mais qui demanderait à être exploré plus tard), c'était le dessin du son, de la parole. C'était un malaxage de paragraphes, de séquences, de phrases, de mots qui, à force d'être déplacés, organisés, réorganisés, me donnaient l'impression que les masses d'écritures que j'avais produites devenaient comme des masses de terre glaise. C'était l'impression que l'écrivain que j'étais, comme le potier ou le sculpteur, façonnait son texte avec ses doigts.

Cela devenait comme une sculpture qui allait parler. Car cette sculpture qui n'était pas tout à fait en trois dimensions (elle ne pouvait se voir que de face) était en mouvement et se déroulait de haut en bas ou de droite à gauche devant moi qui étais immobile. Le son était un mouvement dans l'espace et, par conséquent, dans le temps. Et pour témoigner de ce qui m'arrivait - ou de ce que j'aurais voulu qui arrivât quand je parlais, j'avais besoin d'une carte, d'un emploi du temps, d'un programme, d'une partition qui témoigne d'un mouvement dans l'espace et dans le temps, d'un plan de vol du voyage de parole que j'allais entreprendre et proposer à ceux qui m'accompagneraient. C'est ce que me promettait l'ordinateur.

\section{Plan de vol}

L'inscription numérique s'inscrit verticalement devant moi sur un écran. L'en-haut est toujours plus fini que le bas qui est encore vide et donc en devenir. L'ordinateur me permet de me promener, par son ascenseur, de haut en bas ou de bas en haut, me conduisant, bien plus encore que le livre, obstinément vers la sortie, vers mon but, qui sera obligatoirement en bas.

Et, tandis que je remonte mon curseur de déroulement vers le haut pour revoir le début, je vois mon texte descendre vers le bas comme le fait le contre-poids d'un ascenseur, et j'ai l'impression de monter. Pour avancer dans la vision de mon texte, je fais descendre mon curseur vers le bas, le texte monte et j'ai l'impression de descendre. Il y a entre le texte numérique et moi une alternance de chute et d'ascension qui pourrait se déterminer par la différence d'un poids. Ce sont des haut-le-cœur ou des vertiges permanents. En fait, en écrivant sur l'écran d'un ordinateur, je suis comme le pilote d'un avion qui, en déplaçant son manche d'une manière ou d'une autre par de petits mouvemenets, commande à son appareil de se déplacer dans l'espace. L'informatique témoigne de l'inscription de la littérature dans l'espace.

Avec l'ordinateur, je voyage entre le haut et le bas. Une grande roue, une balançoire entre mon intention et son terme. Il y aurait, dans la lecture numérique bien plus que dans la lecture de l'imprimé, l'idée que 
l'origine du texte se trouve en haut, au-delà de l'écran, " dans le ciel? appelée par une prière? venue d'une inspiration? » Et aussi que la continuité du texte, après que l'écriture ou la lecture soient achevées, un endeçà du texte, un en bas, un trou, un enfer? un autre? une remise en question?... un atterrissage? un effacement?

L'horizontalité, elle, est tout le contraire, en tous cas, très différente du cylindre du parchemin numérique qui se déroule devant moi du haut vers le bas. Comme le battant d'un métronome, c'est une rayure mesurée, sans cesse recommencée. Par sa longueur, elle détermine un souffle allant toujours de gauche à droite et sans jamais dévier de son parallélisme parfait, elle me chasse vers la droite. Et là, la droite, c'est un mur de pelote basque, c'est le rebond. C'est la fin d'une infime partie de mon chemin, et la gauche son recommencement, mon effort, mon pas, mon coup de raquette. Et le prolongement, l'en-deçà ou l'au-delà de cette ligne, c'est un parallèle circulaire que je devine comme faisant le tour du monde pour me ramener par ce long chemin, au début de la ligne suivante.

La verticalité de la page conjuguée à l'horizontalité de la ligne me dessine une orbite spiralée. Ce pourrait être comme les chemins en lacets qui permettent l'ascension d'une très haute montagne. Mais si l'on s'en tient à ce qui se passe sur un écran, les chemins que j'y dessine se construisent et s'empruntent en descendant. En écrivant, je descends.

Pourtant la fin de l'histoire que je suis en train d'écrire ou de lire, son issue, son achèvement, son terme sont aussi son couronnement. Ils sont, dans mon esprit, son sommet. J'ai attendu, j'ai souffert comme le héros de l'histoire pour en arriver là! Et cet effort, cette fatigue, cette tension à emprunter les lacets de l'histoire ne peut être que comparable à une ascension. Mais, sur l'écran de mon ordinateur, je descends. Je monterais donc en descendant. Il y a là une représentation mentale du mouvement de ma pensée et de mon écriture difficile à concevoir.

Peut-être ce mouvement d'ascension descendante s'inscrit-il dans un espace sphérique dans lequel haut et bas seraient partout et toujours face-à-face. Et que, comme traversant la Terre d'un point à son antipode, je serais toujours en train de descendre tant que je n'aurais pas atteint la sortie. Il faudrait impérativement descendre pour atteindre mon sommet. Le geste d'inscrire ou de lire sur un ordinateur, comme finalement dans un livre me pose un problème de représentation de mes mouvements mentaux dans l'espace.

Dans la verticalité, la masse des lettres monte ou descend lorsqu'il y a déjà quantité. Mais dans l'écriture horizontale, c'est la naissance du texte. L'horizontal, c'est l'apparition ou le vide. Un vide qui ne peut être qu'inattendu. Aussi inattendu que l'apparition du son dans un silence.

Je me dis qu'il serait intéressant que la lecture numérique d'œuvre littéraire sur nos futurs parchemins électroniques puisse s'envisager par l'apparition du texte au fur et à mesure de sa découverte, comme il en est lors de son inscription initiale, et comme il en est aussi dans une production de parole dans l'espace sonore. Et si l'on voulait, plus encore, que l'écriture et la lecture numériques témoignent au plus près de ce qu'est la parole, il faudrait, alors, qu'après être apparue un instant sur l'écran, cette écriture disparaisse comme il en est de la parole prononcée. 
Ainsi, dans mon intention de traduire de l'invisible avec du visible et dans cet espace orthogonal avec ces cheminements obligatoires et probablement circulaires, je dessine et je sculpte un temps hypothétique dans un espace provisoire semblable au ciel traversé par un avion à réaction et sa trace. Ce que je vois, ou ce que je désirerais faire voir grâce à l'ordinateur, c'est la matérialité du son de ma parole en mouvement.

Pour le moment, avec le bout de mes doigts commandés par mon œil, je dessine et je sculpte une frise verticale, faite de signes minimaux organisés en rayures, comme le firent depuis des siècles, les écrivains qui nous ont précédés. Mais ce malaxage, cette sculpture préalable au son que permet l'ordinateur me rapproche de ce qui devrait être une partition de paroles, une trace, une carte, un ensemble de signes visibles qui m'aidera à mieux retrouver ce que j'ai à dire et comment.

\section{Un SITE INTERNET AU SERVICE DE L'ORALITÉ}

Je viens d'évoquer, par quelques réflexions, hypothèses de travail ou récits d'expériences, la relation que j'entretiens avec mon ordinateur pour écrire ou inscrire quelque chose qui me rapprocherait de l'oralité. Mais devant l'omniprésence de l'informatique dans tous les domaines de la vie contemporaine, et aussi devant tous les avantages évidents qu'elle nous apporte, il n'est pas inutile de savoir à quelle place nous pouvons l'utiliser entre nous.

Sachant qu'elle aussi, comme le livre l'a fait en son temps, nous rapproche à travers le temps et l'espace, mais que, comme le livre ou le cinéma, elle ne nous permet pas totalement de nous embrasser. Ces deux médias laissent entre nous une feuille de papier, un écran ou une image électronique qualifiée de virtuelle qui font toute la différence. Ce rapprochement qu'ils permettent ne doit être compris que comme un chemin, une approche de la parole vivante.

J'ai eu, en tant que directeur du Centre de Littérature Orale (CliO), devenu aujourd'hui Conservatoire contemporain de Littérature Orale, la responsabilité de participer, avec d'autres, au développement de cette aussi nouvelle qu'ancienne discipline qu'est le conte. Il était évident que la communication électronique devait nous aider à nous faire connaître, à faire connaître les conteurs et le répertoire océanique que constituent tous les récits oraux - et désormais écrits et quelquefois enregistrés - qui ont été collectés dans le monde.

Nous avons commencé au $\mathrm{CliO}$ cette action militante et documentaire sur le réseau en 1996. Cela n'a pas été sans difficultés, sans échecs et désespérances mais, peu à peu, nous avons réussi à mettre en place un site informatif, une ébauche de site documentaire et un lieu dit « artistique ». Il n'y a rien d'original dans la mise en place d'un site d'information. Il faut le tenir à jour, qu'il soit riche et généreux, qu'il corresponde aux vœux et besoins des interlocuteurs que l'on souhaite rassembler et faire en sorte que ceux-ci le découvrent et y restent fidèles. C'est ce que nous avons tenté en l'enrichissant chaque année un peu plus jusqu'à 
obtenir une fréquentation assez honorable de huit cents à mille visiteurs par jour, grâce aux structurations habiles de Florence Davignon. Nous avons adjoint à ce site, sur l'idée de Cécile Cayla-Boucharel et avec son concours, une radio conte, ce qui semblait une évidence dans nos perspectives militantes de promotion du conte, qui offre un catalogue d'auditions possibles de plus de cent récits et extraits et des retransmissions de spectacles et de récitals en temps réel.

Mais, sans négliger la construction et de l'évolution de ce site informatif et d'échanges et le développement de notre radio, c'est vers la création et l'invention d'un site " artistique ", que tous nos efforts doivent tendre. C'est à travers lui que va se dessiner une nouvelle forme de traces de paroles, à travers lui que vont se dessiner de nouvelles formes d'expression sensibles qui donneront, à nos gestes quotidiens et prosaïques de transmission numérique, l'impact, l'ambition et la perfection qui leur manquent. C'est, peut-être, à travers lui, que nous redécouvrirons et ferons redécouvrir le besoin simple et vital de nous rassembler pour entendre, voir, partager et reprendre à notre compte des histoires racontées.

\section{L'ÉCRITURE NUMÉRIQUE AU SERVICE DE LA PAROLE}

Ce qui caractérise formellement l'oralité, c'est le son et tout ce qui en découle. Les Dogons décrivent la parole comme un mariage des éléments : air, terre, eau et feu. Et bien que prenant sa forme finale et sonore dans l'air, elle se meut et se tisse, en amont et en aval, en nous et dans l'autre, de la même manière que ses éléments fondateurs. Elle emprunte leurs mouvements, leurs vitesses, leurs formes puisqu'elle est fabriquée par eux.

C'est pourquoi j'ai d'abord souhaité, avec l'un de nos excellents webmasters, Stéphane Bourriez, accompagner l'émission sonore d'un conte d'images emblématiques de mouvements qui s'inspireraient de celles que produit la circulation des éléments, et plus particulièrement de celle de l'eau. Je voyais là l'occasion d'insister sur l'analogie qui existe entre l'eau et la parole. Mais si la parole est très proche dans ses vertus du liquide, elle se manifeste physiquement dans l'air. Comme l'air qui nous traverse, elle est d'abord aérienne. Elle est respirée. S'est alors imposée à nous la nécessité de témoigner du mouvement du souffle du conteur et du texte par le moyen d'images en mouvement. Cette respiration spécifique à la narration traditionnelle s'articule en syllabes, en mètres, vers, phrases, paragraphes, séquences, parties; elle définit les formes et l'architecture linéaire et temporelle de l'expression parlée. En représentant visuellement les apparitions et disparitions successives et scandées de la parole nous voulions mettre en évidence la forme invisible de la parole et, par ce fait, multiplier l'efficacité de ses mouvements et de son élan. Cette restitution d'images mêlée à une audition correspondante devient alors une sorte de partition qui pourrait servir à l'apprentissage oral d'un conte ou demeurer tout simplement une forme visuelle et sonore qui se suffirait à elle-même. 
Les films étrangers nous offrent simultanément images, musiques et textes des sous-titrages entrelacés. Les interviews de personnes s'exprimant avec difficulté sont eux aussi sous titrés. Les conférences au cours desquelles un orateur accompagne son exposé oral de la vision du texte de ce qu'il est en train de dire, à l'aide du logiciel Power Point, nous posent la question de la nécessité de la présence vivante de l'orateur. Ce qui se passe, en réalité, c'est en effet que nous entendons mieux tout ces parleurs, non pas parce que le discours de ces acteurs, ces bredouillants ou ce conférencier sont incompréhensibles (ils ne le sont jamais tout à fait), mais parce qu'en percevant simultanément par les yeux et par les oreilles, nous comprenons mieux. Ce nouveau langage nous aide à comprendre plus entièrement.

On voit là naître un nouveau langage, qui peut s'enrichir visuellement à l'infini, mais on voit aussi que la simple présence humaine demeure indispensable à une véritable narration, à une véritable rencontre. Sans elle, les objets du message n'offrent pas, aussi perfectionnés soient-ils, cette sensible interprétation de l'instant et de l'autre que seule peut susciter une présence vivante. Ces ajouts demeurent des vêtements sur un mannequin de bois. Et si chacun sait, après avoir lu le conte d'Andersen « Les habits neufs de l'empereur », qu'il ne faut pas porter des vêtements trop invisibles pour ne pas se retrouver nu, il faut savoir aussi qu'il ne faut pas laisser ses vêtements, aussi beaux soient-ils, se promener sans personne dedans pour ne pas se retrouver plus que nu, c'est-à-dire pas du tout.

C'est vers une généralisation sensible de cette simultanéité d'audition et de vision d'images, de musiques, de sons et d'écriture que se situe pour nous l'une des pistes que nous souhaitons explorer dans le site artistique du CliO.

\section{VOYAGER DANS UN MONDE EN MOUVEMENT}

Il me reste encore quelque chose à évoquer avant de vous laisser partir. Ces récits élaborés, dont le but est de faire apparaître l'invisible par du visible, et qui deviennent alors des sculptures, des tissus, des films, deviennent aussi des plans d'espace, des architectures, des maisons, des paysages, des pays, des ciels.

Je n'utilise pas fortuitement ce terme de "maisons ». Je pense à cette technique mnémotechnique antique dite "des maisons » qui permettait à l'orateur, au moyen d'une architecture intérieure imaginaire, de mémoriser ses connaissances. Ce que nous désirons exprimer, avant d'être des paroles ou des textes ou des images, est originellement autre chose, que nous qualifions de pensées. Ce peut être évidemment des images, mais aussi bien d'autres choses, protéiformes ou informelles. Elles nous apparaissent dans des espaces, des rangements, que nous percevons plus ou moins consciemment et que nous avons plus ou moins cultivés.

C'est avec la lunette qu'est la surface d'un écran que l'on peut s'aider à voir, ou à concevoir, les espaces qui nous habitent et les lieux où gisent nos connaissances, nos souvenirs, nos sentiments et nos pensées. 
Saint Augustin décrit, dans ses mémoires, les mouvements de son attention à l'intérieur de sa mémoire. Il décrit cette attention mobile, agile et vive comme le vol d'un oiseau qu'il serait à l'intérieur de luimême. Aujourd'hui nous pourrions nous décrire nous-mêmes avec notre ordinateur comme un aviateur aux commandes de son avion dans le monde imaginaire que nous sommes nous-mêmes. Alors ce qui, pour le moment, n'est qu'un écran devient, de fait, une fenêtre, un hublot, ouvert sur un espace infini qui est celui de notre mémoire. Et ce que nous faisons, lorsque nous inventons ou que nous donnons à explorer une histoire sur un ordinateur ou sur un livre, c'est un déplacement du hublot en même temps que nous mêmes et notre outil de navigation dans l'océan infini des possibles

Il y encore le fait que cet infini est lui aussi en mouvement, tout comme le monde réel dont il est le reflet. Nous sommes en mouvement dans un espace en mouvement et il faut alors apprendre à naviguer, à connaître les vents et les courants, les attractions gravitationnelles. Et cette lunette, ce hublot qui nous montre une infime partie de l'infini nous laisse présager que cet infini virtuel est peut-être l'infini réel dans lequel se trouve cet ordinateur qui nous transporte et que nous sommes censés commander.

Dans les perspectives de voyages dans l'espace que nous offre l'ordinateur d'aujourd'hui, ce qui demeure étonnant et qui semble manquer irrémédiablement dans toutes ses performances, est l'absence d'un lieu véritable. L'ordinateur n'en n'est pas un. Comme tous les objets liés à la communication, il est effacé par l'ailleurs. Lorsque je suis en train de communiquer avec un ordinateur, j'ai accès à tous les instants, à tous les sons, à toutes les paroles, à toutes les musiques, à toutes les images, on pourrait presque dire à tous les temps, à tous les autres, à tous les espaces, mais je n'ai pas accès à un lieu dans lequel tout ces tous deviendraient véritablement vivants. Je ne sais pas où ils sont et, par là, je ne sais pas où je suis. Malgré tous ces liens, je suis seul et j'ai froid. I1 n'y a pas le feu d'un autre pour me réchauffer. Là se trouve une question à laquelle l'ordinateur ne peut pas encore répondre, mais qu'il va s'empresser de résoudre dès demain par le moyen d'un GPs intégré.

Je suis un conteur et je veux raconter des histoires par la parole. Je connais le prix de cette parole, je sais combien d'efforts ont contribué à m'en transmettre l'usage et combien elle permettra aux enfants de demain de se construire, eux et la société humaine qu'ils vont élaborer. Je sais qu'il n'y a rien de mieux et de plus exigeant, mais aussi de plus prometteur, qu'une parole échangée entre deux êtres vivants au même endroit et au même instant et cela l'ordinateur, malgré tout, ne le peut pas tout à fait. Et le permettrait-il qu'il faudrait se demander alors pourquoi il serait tout à coup nécessaire.

Il y aura beaucoup à faire encore et à essayer avant que ce moyen de communication, qui est maintenant le nôtre, n'acquière une solidité, un sérieux et une gravité qui appartiennent encore à ce que nous produisons simplement avec la main ou la bouche.

Nous ne trouverons pas avant longtemps un moyen plus parfait, plus universel et plus démocratique, au grand sens du terme, de 
communication que celui de la parole directe d'homme à homme. C'est une telle évidence qu'elle est trop souvent oubliée. Il ne faudrait pas qu'une invention, aussi révolutionnaire soit-elle, nous cache, au lieu de nous y inviter, la nécessité impérieuse et déterminante de nous rapprocher par la parole les uns des autres. 